Comonotonic modification of random vector in its own probability space

Jan Dhaene; Alexander Kukush

DEPARTMENT OF ACCOUNTANCY, FINANCE AND INSURANCE (AFI) 


\title{
Comonotonic modification of random vector in its own probability space.
}

\author{
Jan Dhaene* $\quad$ Alexander Kukush ${ }^{\ddagger}$
}

January 9, 2011

\begin{abstract}
For any random vector $\underline{X}=\left(X_{1}, \ldots, X_{n}\right)$ on a given probability space $(\Omega, \mathcal{F}, \operatorname{Pr})$, one can always onstruct comonotonic modifications of $\underline{X}$, which are defined as random vectors with the same marginals as $\underline{X}$ but with the comonotonic copula describing their dependency structure. In this short note, we investigate whether it is always possible or not to define comonotonic modifications of $\underline{X}$ on its own probability space $(\Omega, \mathcal{F}, \operatorname{Pr})$.
\end{abstract}

Keywords: Comonotonic random vector, comonotonic modification, non-atomic probability space.

\section{Introduction}

Consider a random vector $\underline{X}=\left(X_{1}, \ldots, X_{n}\right)$ defined on a given probability space $(\Omega, \mathcal{F}, \operatorname{Pr})$. Loosely speaking, the random vector $\underline{X}$ is comonotonic if its multivariate distribution is equal to the multivariate distribution of a random vector $\underline{Y}=\left(Y_{1}, \ldots, Y_{n}\right)$ of which the stochasticity can be captured by a single driver, which means that all random variables $Y_{i}$ behave as nondecreasing functions of a single underlying random variable.

Let us now assume that $\underline{X}$ is not comonotonic. Then one can always construct a comonotonic modification of $\underline{X}$, that is a comonotonic random vector $\underline{X}^{c}=\left(X_{1}^{c}, \ldots, X_{n}^{c}\right)$ which has the same marginal distributions as $\underline{X}$.

The probability space $(\Omega, \mathcal{F}, \operatorname{Pr})$ on which $\underline{X}$ is defined may or may not be rich enough to contain comonotonic modifications of $\underline{X}$. In case it is not rich enough, the comonotonic modifications $\underline{X}^{c}$ will necessary have to be defined on another probability space.

In this short note we present a condition on the probability space, under which comonotonic modifications can always be constructed on the same probability space as the one of the original random vector $\underline{X}$. We also give a simple example of probability space containing a random vector $\underline{X}$ for which no comonotonic modification can be constructed without leaving the original space.

This note is structured as follows. In Section 2 we briefly describe the concept of comonotonicity and some of its properties. The existence of the comonotonic modification of a random vector on its own probability space is discussed in Section 3. Finally, Section 4 concludes the paper.

\section{Comonotonic random variables}

In the sequel, a $n$-vector $\left(x_{1}, \ldots, x_{n}\right)$ will often be denoted by $\underline{x}$. For two $n$-vectors $\underline{x}$ and $\underline{y}$ the notation $\underline{x} \leq \underline{y}$ is used for the componentwise order which is defined by $x_{i} \leq y_{i}$ for all $i=1, \ldots, n$.

\footnotetext{
*FBE, K.U.Leuven, Naamsestraat 69, B-3000 Leuven, Belgium, jan.dhaene@econ.kuleuven.be.

${ }^{\dagger}$ Faculty of Mechanics and Mathematics, National Taras Shevchenko University of Kyiv, Volodymyrska st. 64, 01601 Kyiv, Ukraine, alexander_kukush@univ.kiev.ua.

${ }^{\ddagger}$ Corresponding author. Tel. $+38-044-2792634$, Fax $+38-044-2661558$.
} 
Definition 1 A set $A \subseteq \mathbb{R}^{n}$ is said to be comonotonic if for any $\underline{x}$ and $\underline{y}$ in $A$, either $\underline{x} \leq \underline{y}$ or $\underline{y} \leq \underline{x}$ holds.

Hereafter we restate the definition of the notion of comonotonicity of random vectors, as presented in Kaas et al. (2000).

Definition $2 A$ random vector $\underline{X}$ is said to be comonotonic if it has a comonotonic support.

In the following theorem, some characterizations are given for the comonotonicity of a random vectors. The notation $F_{X}^{-1}$ is used for the usual inverse of the distribution function $F_{X}$ of the r.v. $X$, i.e.

$$
F_{X_{i}}^{-1}(p)=\inf \left\{x \in \mathbb{R} \mid F_{X_{i}}(x) \geq p\right\}, \quad p \in[0,1]
$$

with inf $\varnothing=+\infty$ by convention. Furthermore, in the remainder of this note, the notation $U$ is used to denote a r.v. which is uniformly distributed on the unit interval $(0,1)$. Finally, the notation $\stackrel{d}{=}$ is used to denote 'equality in distribution'.

Theorem 3 A random vector $\underline{X}=\left(X_{1}, \ldots, X_{n}\right)$ is comonotonic if, and only if, one of the following equivalent conditions holds:

(1) For $U \sim$ Uniform $(0,1)$, we have that

$$
\underline{X} \stackrel{d}{=}\left(F_{X_{1}}^{-1}(U), \ldots, F_{X_{n}}^{-1}(U)\right) .
$$

(2) There exists a r.v. $Z$ and non-decreasing functions $f_{i}: \mathbb{R} \rightarrow \mathbb{R}, i=1, \ldots, n$, such that

$$
\underline{X} \stackrel{d}{=}\left(f_{1}(Z), \ldots, f_{n}(Z)\right) \text {. }
$$

For a proof of this theorem, see e.g. Denneberg (1997). An overview of the properties of comonotonic random vectors can be found in Dhaene et al. (2002a). An overview of actuarial and financial applications of the concept of comonotonicity is presented in Dhaene et al. (2002b) and Deelstra et al. (2011).

\section{Comonotonic modifications of a random vector in its own probability space}

In this section we investigate conditions under which for any random vector $\underline{X}=\left(X_{1}, X_{2}, \ldots, X_{n}\right)$ defined on a given probability space $(\Omega, \mathcal{F}, \operatorname{Pr})$, one can construct a comonotonic modification on this same probability space.

Definition 4 A random vector $\underline{Y}$ is said to be a comonotonic modification of the random vector $\underline{X}$ if $\underline{Y}$ is comonotonic and has the same marginal distributions as $\underline{X}$.

For any random vector $\underline{X}$ defined on a probability space $(\Omega, \mathcal{F}, \operatorname{Pr})$ one can construct comonotonic modifications. Indeed, for any $U \sim \operatorname{Uniform}(0,1)$, we have that

$\left(F_{X_{1}}^{-1}(U), \ldots, F_{X_{n}}^{-1}(U)\right)$ is a comonotonic modification of $\underline{X}$. Notice that $U$ and hence also the comonotonic modification $\left(F_{X_{1}}^{-1}(U), \ldots, F_{X_{n}}^{-1}(U)\right)$ of $\underline{X}$ are not necessarily defined on $(\Omega, \mathcal{F}, \operatorname{Pr})$.

Before we introduce a condition which guarantees the existence of comontonic modifications of a random vector on its own probability space, we first consider a condition guaranteeing the existence of uniformly distributed r.v.'s on a given probability space. The notion of 'non-atomic probability spaces' turns out to be essential in that respect. 
Definition 5 A probability space $(\Omega, \mathcal{F}, \operatorname{Pr})$ is said to be atomic if there exists an event $B \in \mathcal{F}$ with $\operatorname{Pr}[B]>0$, for which it is impossible to find another event $A \subset B$ such that $0<\operatorname{Pr}[A]<$ $\operatorname{Pr}[B]$.

The event $B$ in the definition above is called an atom of the probability space under consideration. Intuitively, an atom of a given probability space is a non-zero event which cannot be divided in nontrivial sub-events.

A probability space without atoms is called a non-atomic space. The space $\left([0,1], \mathcal{F}_{[0,1]}, \lambda\right)$, with $\mathcal{F}_{[0,1]}$ being the class of all Lebesgue measurable subsets of $[0,1]$ and $\lambda$ the Lebesgue measure, is an example of a non-atomic probability space.

In a discrete probability space, i.e. a probability space with a countable (finite or countably infinite) universe, any $\omega \in \Omega$ with $\operatorname{Pr}[\{\omega\}]>0$ is an atom.

Another example of an atomic probabily space is $([0,1], \mathcal{F}, \mu)$. Here $\mathcal{F}$ is the set of all finite or countable subsets $A$ of the unit interval $[0,1]$ as well as their complements $[0,1] \backslash A$. Hence, $\mathcal{F}=\{A,[0,1] \backslash A \mid A \subseteq[0,1]$ is finite or countable $\}$. Further, for any finite our countable $A \subseteq$ $[0,1]$, we set $\mu[A]=0$ and $\mu[[0,1] \backslash A]=1$. It follows immediately that any $[0,1] \backslash A$ is an atom of the probability space under consideration.

In the following two lemmas, we prove that a probability space contains uniformly distributed, and more generally continuously distributed r.v.'s, if and only if this space is non-atomic.

Lemma 6 On a given probability space $(\Omega, \mathcal{F}, \operatorname{Pr})$, one can construct a r.v. $U \sim$ Uniform $(0,1)$ if, and only if, this space is non-atomic.

Proof. First, let the space $(\Omega, \mathcal{F}, \operatorname{Pr})$ have atoms, i.e., there exists an event $B$ with $\operatorname{Pr}[B]>0$ such that all measurable subsets of $B$ have probability either 0 or $\operatorname{Pr}[B]$. Then for any r.v. $X$ defined on $(\Omega, \mathcal{F}, \operatorname{Pr})$, we must have that $\operatorname{Pr}[X \leq x$ and $B]$ equals either 0 or $\operatorname{Pr}[B]$. This implies that $\operatorname{Pr}[X \leq x \mid B]$ can only take the values 0 or 1 . Hence, there exist a real number $x_{0}$ such that $\operatorname{Pr}\left[X=x_{0} \mid B\right]=1$. From this observation we find that $\operatorname{Pr}\left[X=x_{0}\right] \geq \operatorname{Pr}\left[X=x_{0}\right.$ and $\left.B\right]=$ $\operatorname{Pr}[B]>0$, which implies that $X$ cannot have a continuous cdf. In particular, $X$ can not be uniformly distributed on the unit interval.

Now let $(\Omega, \mathcal{F}, \operatorname{Pr})$ be non-atomic. Then for each number $n$, we can divide $\Omega$ into disjoint parts $\Omega_{i n}, i=1, \ldots, n$, with $\operatorname{Pr}\left(\Omega_{i n}\right)=\frac{1}{n}, i=1, \ldots, n$, see Corollary 1.12.10 in Bogachev (2007). Introduce the r.v.'s $U_{n}$ by $U_{n}(\omega)=\frac{i}{n}, \omega \in \Omega_{i n}, i=1, \ldots, n$. The sequence of probability laws $L\left(U_{n}\right)$ weakly converges to the Uniform $(0,1)$ law. Then from Engl \& Wakolbienger (1983) it follows that there exists a r.v. $U$ on $(\Omega, \mathcal{F}, \operatorname{Pr})$, with a Uniform $(0,1)$ distributed limit distribution.

Lemma 6 immediately leads to the following result.

Lemma 7 On a given probability space $(\Omega, \mathcal{F}, \operatorname{Pr})$, one can define r.v.'s with a continuous cdf if, and only if, this space is non-atomic.

Proof. From Lemma 6 we know that a non-atomic space contains r.v.'s $U \sim$ Uniform $(0,1)$, which have a continuous cdf.

On the other hand, if $(\Omega, \mathcal{F}, \operatorname{Pr})$ contains a r.v. $X$ with a continuous cdf, then also $F_{X}(X) \sim$ Uniform $(0,1)$ is a r.v. on this probability. From Lemma 6 we can conclude that this space is non-atomic.

From Lemma 6, we find the following corollary.

Corollary 8 For any random vector $\underline{X}$ which is defined on a non-atomic probability space, one can construct a comonotonic modification which is defined on the same probability space. 
Proof. As $\underline{X}$ is defined on a non-atomic probability space $(\Omega, \mathcal{F}, \operatorname{Pr})$, we find from Lemma 6 that there exists a r.v. $U \sim$ Uniform $(0,1)$ defined on $(\Omega, \mathcal{F}, \operatorname{Pr})$. The proof follows then from the fact that the random vector $\left(F_{X_{1}}^{-1}(U), \ldots, F_{X_{n}}^{-1}(U)\right)$ is a comonotonic modification of $\underline{X}$ defined on $(\Omega, \mathcal{F}, \operatorname{Pr})$.

Consider the special case of a random vector $\underline{X}=\left(X_{1}, \ldots, X_{n}\right)$ defined on $(\Omega, \mathcal{F}, \operatorname{Pr})$, where at least one of the $X_{i}$ has a continuous cdf. From Lemma 7 , we know that $(\Omega, \mathcal{F}, \operatorname{Pr})$ is nonatomic. From Corollary 8 we can conclude that there exists a comonotonic modification of $\underline{X}$ on $(\Omega, \mathcal{F}, \operatorname{Pr})$. In this particular case, it is straightforward to construct such a comonotonic modification. Without loss of generality, we may assume that $X_{1}$ has a continuous cdf. Then $F_{X_{1}}\left(X_{1}\right) \sim \operatorname{Uniform}(0,1)$ and the random vector $\underline{X}^{c}$ defined by

$$
\underline{X}^{c}=\left(X_{1}, F_{X_{2}}^{-1}\left(F_{X_{1}}\left(X_{1}\right)\right), \ldots, F_{X_{n}}^{-1}\left(F_{X_{1}}\left(X_{1}\right)\right)\right)
$$

is a comonotonic modification of $\underline{X}$ defined on $(\Omega, \mathcal{F}, \operatorname{Pr})$.

Let us now consider the case of a random vector defined on a given atomic probability space and investigate whether it is possible or not to construct comonotonic modification of that random vector on the same probability space.

Example 9 Consider the probability space $(\Omega, \mathcal{F}, \operatorname{Pr})$. The universe is given by $\Omega=\left\{\omega_{1}, \omega_{2}\right\}$. Further, $\mathcal{F}$ is the $\sigma$-field of all subsets of $\Omega$. Finally, $\operatorname{Pr}$ is the probability measure on $(\Omega, \mathcal{F})$ defined by

$$
0<\operatorname{Pr}\left[\left\{\omega_{1}\right\}\right]=p<1 \text { and } \operatorname{Pr}\left[\left\{\omega_{2}\right\}\right]=q=1-p<p .
$$

The random vector $\underline{X}=\left(X_{1}, X_{2}\right)$ defined on $(\Omega, \mathcal{F}, \operatorname{Pr})$ is given by

$$
\underline{X}\left(\omega_{1}\right)=(1,0) \text { and } \underline{X}\left(\omega_{2}\right)=(0,1) .
$$

The marginal distributions of $\underline{X}$ follow from

$$
\operatorname{Pr}\left[X_{1}=0\right]=q=1-\operatorname{Pr}\left[X_{1}=1\right]
$$

and

$$
\operatorname{Pr}\left[X_{2}=0\right]=p=1-\operatorname{Pr}\left[X_{2}=1\right] .
$$

The quantile functions of $X_{1}$ and $X_{2}$ are given by

$$
F_{X_{1}}^{-1}(u)=\left\{\begin{array}{ll}
0 & : 0<u \leq q \\
1 & : q<u \leq 1
\end{array} \text { and } F_{X_{2}}^{-1}(u)=\left\{\begin{array}{cc}
0 & : 0<u \leq p \\
1 & : p<u \leq 1
\end{array}\right.\right.
$$

For any comonotonic modification $\underline{X}^{c}$ of $\underline{X}$ it holds that

$$
\left.\underline{X}^{c} \stackrel{d}{=}\left(F_{X_{1}}^{-1} U\right), F_{X_{2}}^{-1}(U)\right) \text {. }
$$

From this equality in distribution and the expressions (8) of the quantile functions we find that

$$
\operatorname{Pr}\left[\underline{X}^{c}=(0,0)\right]=q, \operatorname{Pr}\left[\underline{X}^{c}=(1,0)\right]=p-q \text { and } \operatorname{Pr}\left[\underline{X}^{c}=(1,1)\right]=q .
$$

As the probability space $(\Omega, \mathcal{F}, \operatorname{Pr})$ is not rich enough in the sense that it contains no event with probability $p-q$, we can conclude that it is impossible to define a comonotonic modification $\underline{X}^{c}$ of $\underline{X}$ on $(\Omega, \mathcal{F}, \operatorname{Pr})$. 
From the example above we can conclude that in atomic spaces random vectors do not always have comonotonic modifications in that same space. However, the following example proves that there exist atomic probability spaces with random vectors for which one can construct comonotonic modifications without leaving this space.

Example 10 Let the universe be given by $\Omega=\left\{\omega_{1}, \omega_{2}, \omega_{3}\right\}$, while $\mathcal{F}$ is the $\sigma$-field of all subsets of $\Omega$, and $\operatorname{Pr}$ is the probability measure on $(\Omega, \mathcal{F})$ defined by

$$
\left.0<\operatorname{Pr}\left[\left\{\omega_{1}\right\}\right]=q=1-p<p, \operatorname{Pr}\left[\left\{\omega_{2}\right\}\right]=p-q \text { and } \operatorname{Pr}\left[\left\{\omega_{3}\right\}\right]\right)=q .
$$

The random vector $\underline{X}=\left(X_{1}, X_{2}\right)$ on $(\Omega, \mathcal{F}, \operatorname{Pr})$ is defined by

$$
\underline{X}\left(\omega_{1}\right)=\underline{X}\left(\omega_{2}\right)=(1,0) \text { and } \underline{X}\left(\omega_{3}\right)=(0,1) .
$$

As before, the marginal distributions of $\underline{X}$ follow from (6) and (7), while the quantile functions are given by (8). The distribution of any comonotonic modification $\underline{X}^{c}$ of $\underline{X}$ is again given by (10).

Let us now define the random vector $\underline{Y}$ on $(\Omega, \mathcal{F}, \operatorname{Pr})$ by

$$
\underline{Y}\left(\omega_{1}\right)=(0,0), \underline{Y}\left(\omega_{2}\right)=(1,0) \text { and } \underline{Y}\left(\omega_{3}\right)=(1,1) .
$$

As $\underline{Y}$ has a comonotonic support, it is a comonotonic random vector. One can easily verify that $\underline{Y}$ has the same marginal distributions as $\underline{X}$. We can conclude that $\underline{Y}$ is a comonotonic modification of $\underline{X}$, defined on the same probability space as $\underline{X}$.

From the two examples above we find that random vectors defined on an atomic probability space may or may not have a comonotonic modification in that same space.

\section{Conclusions}

In this note, we showed that for a random vector on a probability space without atoms, there always exists a comonotonic modification defined on the same probability space. On the other hand, random vectors defined on an atomic probability space may or may not have a comonotonic modification, depending on the richness of that space.

Acknowledgement. The authors acknowledge the financial support of the Onderzoeksfonds K.U. Leuven (GOA/0\%: Risk Modeling and Valuation of Insurance and Financial Cash Flows, with Applications to Pricing, Provisioning and Solvency).

\section{References}

[1] Bogachev, V.I., 2007. Measure Theory, vol. 1. Springer-Verlag, Berlin, pp. 500.

[2] Deelstra, G., Dhaene, J., Vanmaele, M., 2011. An overview of comonotonicity and its applications in finance ans insurance, in : Di Nunno, B. Øksendal (Eds.), Advanced Mathematical Methods for Finance, Springer.

[3] Denneberg, D., 1997. Non-additive Measure and Integral, 2nd ed. Kluwer Academic Publishers, pp. 184.

[4] Dhaene, J., Denuit, M., Goovaerts, M.J., Kaas, R., Vyncke, D., 2002a. The concept of comonotonicity in actuarial science and finance: theory. Insurance: Mathematics and Economics 31, 3-33. 
[5] Dhaene, J., Denuit, M., Goovaerts, M.J., Kaas, R., Vyncke, D., 2002b. The concept of comonotonicity in actuarial science and finance: applications. Insurance: Mathematics and Economics 31, 133-161.

[6] Engl, H., Wakolbienger, A., 1983. On weak limits of probability distributions on polish spaces. Stochastic Analysis and Applications 1, 197-203.

[7] Kaas, R., Dhaene, J., Goovaerts, M., 2000. Upper and lower bounds for sums of random variables. Insurance: Mathematics and Economics 27(2), 151-168. 